\title{
A large-area AgNW-modified textile with high-performance electromagnetic interference shielding
}

\author{
Junchen Liu ${ }^{1,2}$, Sen Lin ${ }^{1}$, Kai Huang ${ }^{1}$, Chao $\mathrm{Jia}^{2}$, Qingmin Wang ${ }^{1}$, Ziwei Li ${ }^{2}$, Jianan Song ${ }^{2}$, Zhenglian Liu ${ }^{2}$, Haiyang Wang ${ }^{2}$, \\ Ming Lei $\mathbb{D}^{1 凶}$ and Hui Wu iD $^{2 凶}$
}

Manufacturing a flexible, light, large-area, and high-efficiency electromagnetic shielding materials in a straightforward and costeffective manner presently remains a significant challenge. In this work, we propose a conductive network design and verify its electromagnetic interference (EMI) shielding effectiveness (SE) by simulation. Using the structure and parameters obtained by simulation, we prepare a flexible EMI shielding material using silver nanowires (AgNWs)/polyvinyl butyral (PVB) ethanol solution and textile substructure via a facile immersing method. In the frequency range of $5-18 \mathrm{GHz}$, the AgNWs/PVB textile with $1.4 \mathrm{~mm}$ thickness achieves an EMI SE of $59 \mathrm{~dB}$, which exceeds the requirements for commercial applications. Due to the low density of $56 \mathrm{mg} / \mathrm{cm}^{3}$, specific shielding effectiveness (SSE) of this material reaches $1053 \mathrm{~dB} \mathrm{~m} / \mathrm{g}$. It is found that the AgNWs/PVB textile is more resistant to washing with water and oxidation than AgNWs textile without a PVB protector. As a result, the conductivity of AgNWs/PVB textile exhibits no change after washing with water and varies slightly after being kept in hot air. We find that a signal monitor is unable to detect a signal emitted by a mobile phone from a jacket lined with AgNWs/PVB textile. AgNWs/PVB textile with these properties can be mass-produced as high-efficiency EMI shielding material for commercial applications.

npj Flexible Electronics (2020)4:10; https://doi.org/10.1038/s41528-020-0074-0

\section{INTRODUCTION}

With the development of wireless-telecommunication technology, the human environment is becoming increasingly congested with electromagnetic radiation, which may be a health hazard ${ }^{1-3}$. In particular, X-Band electromagnetic with a frequency from 8 to $12 \mathrm{GHz}$ play an important role and are frequently used in military and telecommunications devices ${ }^{4-6}$. In addition, certain high-end precision scientific and military equipment require environment without electromagnetic interference ${ }^{7,8}$. EMl shielding is most commonly based on electromagnetic wave reflection by charge carriers. Therefore, high electrical conductivity is required for EMI shielding materials ${ }^{9,10}$. Electrical conductors such as copper, silver, and carbon-based materials are widely used for EMI shielding ${ }^{11-17}$. However, due to skin effect, the thickness of the EMI SE material cannot be too low, which means that pure metal or carbon-based materials are not suitable for practical use ${ }^{18,19}$.

Along with high EMI SE, low weight, flexibility, and mass production are also essential for EMI shielding materials in practical applications ${ }^{20-23}$. Loading conductive nanomaterials on a light and flexible substructure is a promising approach for preparation of light, flexible, and high-efficiency EMI shielding materials $^{24-27}$. For example conductive nanomaterials can be uniformly dispersed and integrated on the surface of a flexible substrate. Although this procedure is straightforward on a $2 \mathrm{D}$ plane, certain issues can appear when increasing the thickness of the conductive 3D structure in order to improve the EMI SE ${ }^{28,29}$. For example, certain nanomaterials cannot enter and adhere on a $3 D$ skeleton or are not sufficiently compatible with the 3D structure.

1D metal nanomaterials are used in flexible electronic materials and devices because they have a large length-diameter ratio and can form a $2 \mathrm{D}$ or $3 \mathrm{D}$ network on various substrates ${ }^{30}$, e.g., silver nanowires (AgNWs), silver nanofibers, copper nanowires and gold nanowires ${ }^{31-35}$. Silver is more stable than copper and more affordable than Au, making 1D silver nanomaterial (AgNWs) and silver nanofibers commonly used for EMI SE. However, the nanofibers are always collected on a plane and are difficult to integrate into a 3D substrate. In order to increase the thickness of EMI SE materials while preserving flexibility, AgNWs can be loaded on a flexible substrate with $3 D$ structure ${ }^{36-38}$. Many research papers have reported materials with high EMI SE, however, the methods proposed are difficult to scale for the requirements of large markets.

In this work, by using numerical simulations, we find a conductor with a network structure that can reach high EMI SE with a conductivity five orders of magnitude below that of pure silver. To verify the simulation results in practice, we deposit a few AgNWs deposited by a simple dropping method on a type of cotton textile with a network structure. This EMI shielding material does not only achieve a high EMI SE with a very small amount of silver but is also lightweight and flexible. Moreover, a large-area EMI SE cloth can be produced in this way. Herein, we placed the EMI shielding textile in a jacket and the signal of the in-jacket mobile phone couldn't be detected by a signal detector, demonstrating the high EMI SE of this material. In addition, it remains conductive after immersion in water and oxidation. Consequently, we propose this material for applications requiring a light and flexible EMI shielding material.

\section{RESULTS}

Simulation of EMI shielding effectiveness of the network structure According to theory, EMI shielding effect decreases greatly with thickness below skin depth. In order to determine the exact

\footnotetext{
${ }^{1}$ State Key Laboratory of Information Photonics and Optical Communications and School of Science, Beijing University of Posts and Telecommunications, 100876 Beijing, China. ${ }^{2}$ State Key Laboratory of New Ceramics and Fine Processing, School of Materials Science and Engineering, Tsinghua University, 100084 Beijing, China. ${ }^{凶}$ email: mlei@bupt.edu.cn; huiwu@tsinghua.edu.cn
} 

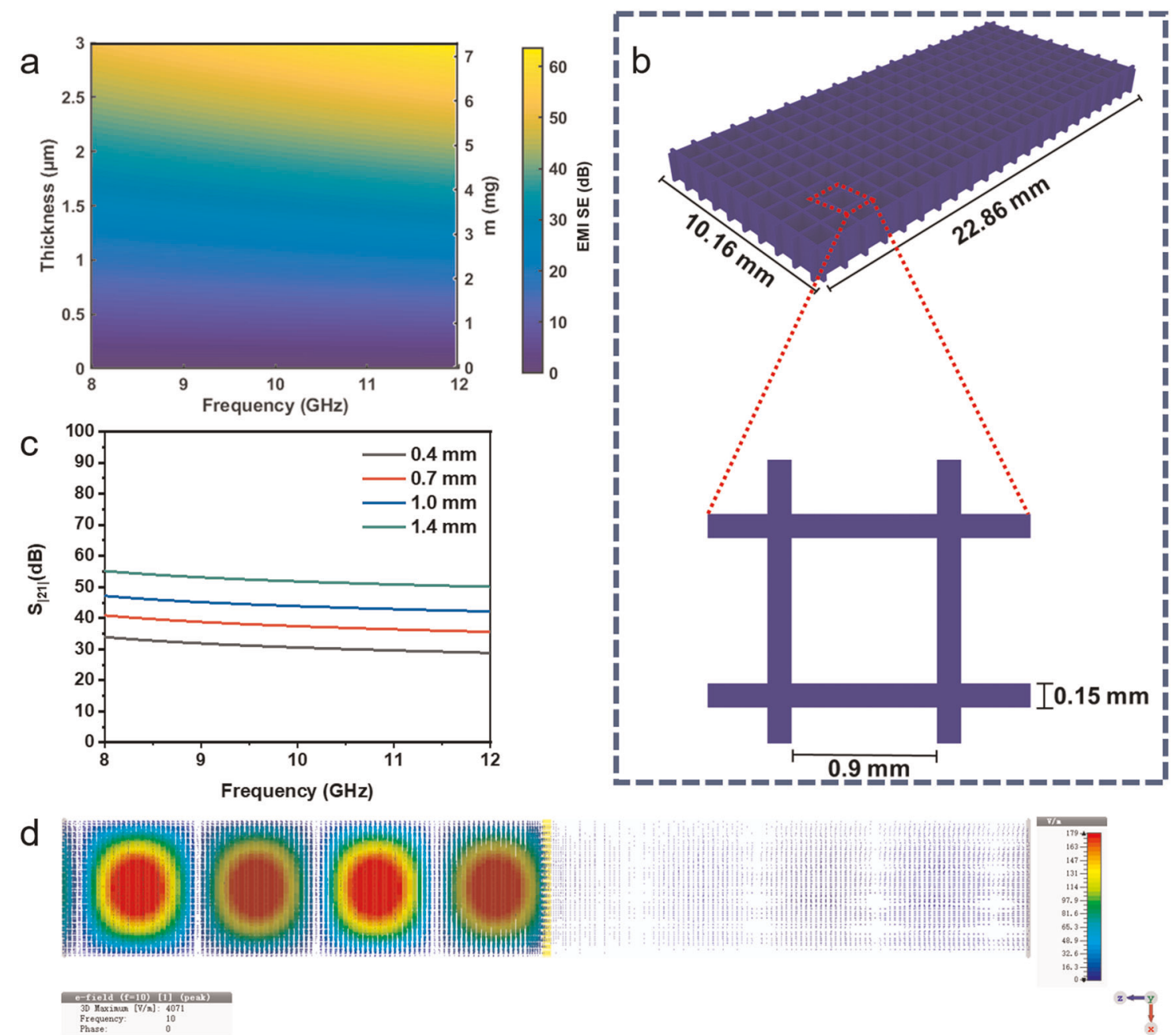

Fig. 1 Simulation and calculation. a Calculation based on the plane wave theory. The dependence of EMI SE of silver plates on thickness and frequency is shown in color. The " $\mathrm{m}$ " label on the right axis denotes the mass calculated with the density of the silver and the surface area of the 8-12 GHz waveguide taken into account. b Model used in the simulation with a network structure unit enlarged. c The result of simulation for various thicknesses. d Electromagnetic field intensity distribution at $10 \mathrm{GHz}$.

relationship between the thickness and EMI SE, we calculate this relationship for silver by using the equation from Supplementary Note. In addition, to visually determine the consumption of silver, we convert the thickness to mass by using the density of silver and the cross-sectional area of an X-band waveguide. It can be seen from Fig. 1a that the EMI SE of a silver plate can reach $60 \mathrm{~dB}$ at $12 \mathrm{GHz}$ with a thickness of $3 \mu \mathrm{m}$. On the other hand, a silver plate of this thickness in an X-Band waveguide contains about $7 \mathrm{mg}$ of silver, making this design insufficiently affordable and flexible. Moreover, such a silver plate is cumbersome to use across a large surface area in practice. Herein, we design a conductor model in a $22.86 \mathrm{~mm} \times 10.16 \mathrm{~mm}$ network structure, which matches a square waveguide for $8-12 \mathrm{GHz}$ frequency (Fig. 1b). Each unit of the network structure is a square frame with an interior side length of $0.9 \mathrm{~mm}$ and width of $0.15 \mathrm{~mm}$. The conductivity of the frame is $630 \mathrm{~S} / \mathrm{m}$, which is five orders of magnitude lower than pure silver. We simulate the EMI SE of this model with different thickness in a square waveguide for frequencies in the $8-12 \mathrm{GHz}$ range. Figure $1 \mathrm{c}$ shows the results of this simulation. We find that S-parameter of $\mathrm{S}_{21}$ of this network structure can reach $35 \mathrm{~dB}$ for $0.4 \mathrm{~mm}$ thickness and $55 \mathrm{~dB}$ for $1.4 \mathrm{~mm}$, showing that a conductor with low conductivity can be an excellent EMI shielding material if shaped into a network. In addition, we monitored the electric field distribution at $10 \mathrm{GHz}$
(Fig. 1d). Electromagnetic waves emitted from left are obstructed by the conductive network structure placed at the middle of the square waveguide. Consequently, a very diminished electromagnetic field is observed at the other side of the EMI shielding material.

Preparation of the network structure for EMI shielding material After obtaining the parameters for the shielding material from the simulation described above, we proceed to prepare a physical sample and investigate it experimentally. One of the most promising approaches is conductive nanomaterials integration on existing flexible and light network structures. We select a type of textile made of cotton due to its lightness, flexibility and cost effectiveness, as well as network structure which matches the one used in the simulation (Supplementary Fig. 1). Its unique cotton fiber skeleton is also advantageous with conductive 1D nanomaterials with high length-diameter ratio that adhere on it. We select AgNWs as the nano-conductive material, since its high conductivity and length-diameter ratio provides electronic access. Moreover, its high chemical stability and cost-effectiveness in comparison with noble metals enable a wide range of future applications (Supplementary Fig. 2). Figure 2a shows the methods we use to prepare large area EMI shielding textile. We immerge the textile in AgNWs/PVB ethanol solution and then dry it. In this way, we 
a

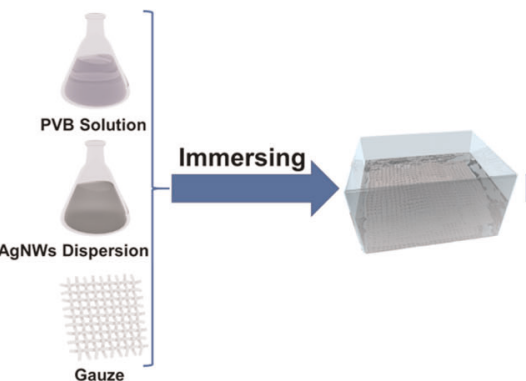

C

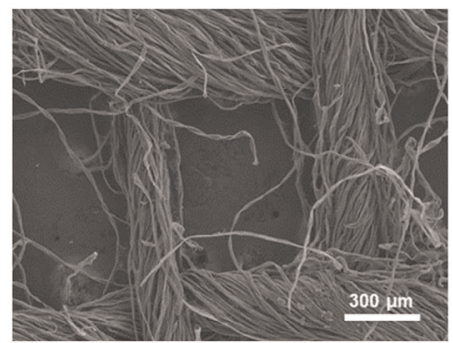

f

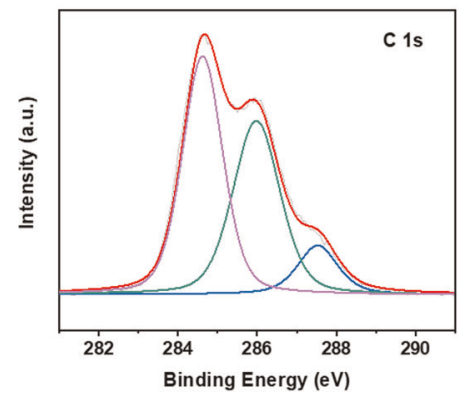

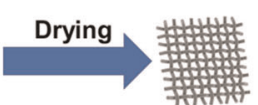

b

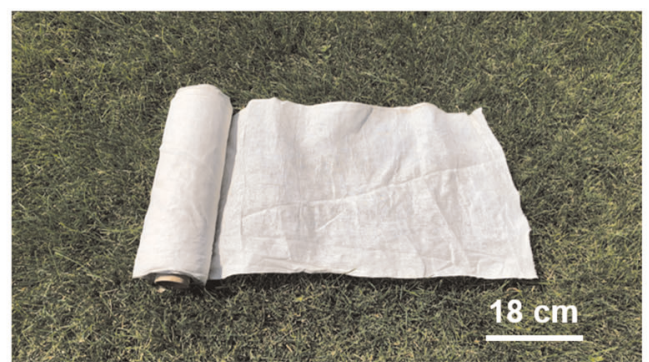

d
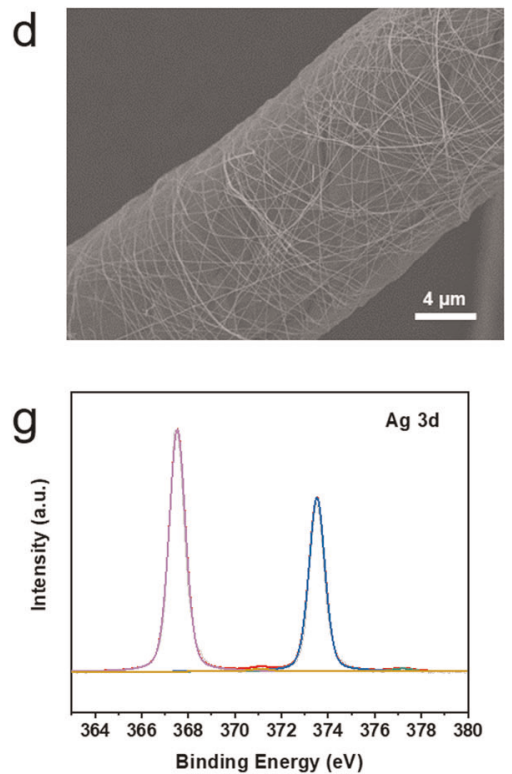

e
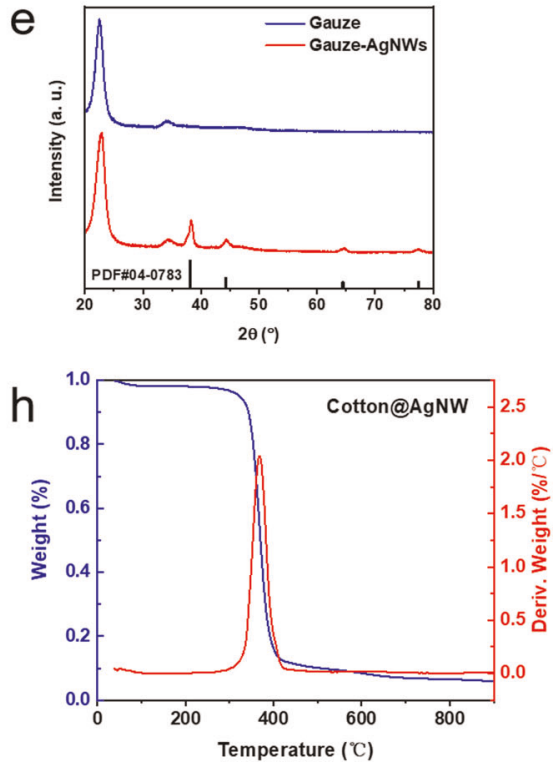

Fig. 2 The preparation process for AgNWs/PVB textile and properties of materials. a AgNWs/PVB textile preparation process schematic. b Photograph of a roll of AgNWs/PVB textile. c SEM image of a unit cell of the AgNWs/PVB textile. d SEM image of a single skeleton of the AgNWs/PVB textile. e XRD spectrum of AgNWs/PVB and pure textile. Chemical bond analysis of the surface of AgNWs/PVB textile using XPS, including peak-differentiation-imitating analysis of $\mathbf{f} \mathrm{C} 1 \mathrm{~s}$ and $\mathbf{g} \mathrm{Ag} 3 \mathrm{~d}$. $\mathbf{h}$ TGA (blue) and DSC (red) results for AgNWs/PVB textile from room temperature to $900^{\circ} \mathrm{C}$.

produce a piece of conductive textile with a conductivity of about $600 \mathrm{~S} / \mathrm{m}$, conforming to simulation parameters. With a density of $56 \mathrm{mg} / \mathrm{cm}^{3}$, it is light and flexible, benefiting from the large dutycycle of the flexible textile substrate as well as the high length-todiameter ratio of AgNWs. A roll of EMI SE textile is shown in Fig. $2 \mathrm{~b}$, c shows the scanning electron microscope (SEM) picture of an AgNWs/PVB textile unit cell, which retains the initial textile network structure prior to immersion processing. Unlike the initial textile, we see a marked change in the SEM picture of the AgNWs/ PVB textile's cotton skeleton. Figure $2 d$ shows a single cotton skeleton on which many AgNWs are distributed and interconnected, which is the reason the AgNWs/PVB is a conductor. Figure 2e shows the X-Ray diffraction (XRD) of textile and AgNWs/PVB textile, showing that after being processed by AgNWs/PVB ethanol solution, apart from the peaks of the textile, the XRD peaks of $\mathrm{Ag}$ can also be detected ${ }^{39}$. The X-ray photoelectron spectroscopy (XPS) scan has also been used to investigate the elements $C$ and Ag. Within the peak of $\mathrm{C} 1 \mathrm{~s}$, three separate peaks can be discerned: unoxidized carbon (C-C or $\mathrm{C}-\mathrm{H}$ at $285.0 \mathrm{eV})$, carbon with one oxygen bond $(\mathrm{C}-\mathrm{OH}$ or $\mathrm{C}-\mathrm{O}-$ at $286.6 \mathrm{eV})$ and carbon with two oxygen bonds (O-C-O or $\mathrm{C}=\mathrm{O}$, respectively (Fig. $2 \mathrm{~g}))^{40}$. The peak of Ag3d consists of two metal peaks at $367.5 \mathrm{eV}$ and $373.5 \mathrm{eV}$, and two tiny featureless peaks at 371.1 and $377.2 \mathrm{eV}$ which correspond to all metallic Ag peaks (Fig. 2g). We also investigate the thermogravimetric (TG) properties of AgNWs/PVB textile (Fig. 2h) and pure textile (Supplementary Fig. 3) in air with the temperature varying from room temperature to $900^{\circ} \mathrm{C}$ and find that both samples exhibit a large decrease at $350^{\circ} \mathrm{C}$. A negligible difference between the samples is seen after the process. The residue of AgNWs/textile exceeds that of pure textile by $3 \%$. Since the temperature is below the vaporization temperature of $\mathrm{Ag}$, we can roughly estimate that the mass fraction of silver in the AgNWs/PVB textile is no more than 3\%. The consumption of silver in AgNWs/PVB textile prepared by this method does not exceed that of a silver plate.

\section{EMI SE of AgNWs/PVB textile}

We measured the EMI SE effect of the as-prepared AgNWs/PVB textile by using a vector network analyzer in $S_{11}$ and $S_{21}$ channels. First, we scan $S_{21}$ channel with samples of different thickness in square waveguides of $5-8,8-12$, and $12-18 \mathrm{GHz}$. Figure $3 a-c$ show that samples with thickness of 0.4 and $1.4 \mathrm{~mm}$ reach $\sim 25$ and $50-55 \mathrm{~dB}$, respectively, which is a remarkable EMI SE effect. Particularly, in the frequency range of $8-12 \mathrm{GHz}$, the EMI SE effect of the $0.4 \mathrm{~mm}$ and $1.4 \mathrm{~mm}$ thick samples is $\sim 30$ and $59 \mathrm{~dB}$, respectively. The experimental results are found to be in good agreement with the simulation. Moreover, the materials with low density (about $56 \mathrm{mg} / \mathrm{cm}^{3}$ ) have a specific EMI SE of $1053 \mathrm{~dB} \mathrm{~m} / \mathrm{g}$ or an SSE/d of $7521 \mathrm{~dB} \mathrm{~cm} / \mathrm{g}$. A table of various EMI SEs is shown in Supplementary Table section and it can be seen that our EMI SE material compares favorably in both SSE and SSE/d with other listed materials. Figure $3 \mathrm{~d}-\mathrm{f}$ shows the $\mathrm{S} 11$ and $\mathrm{S} 21$ of a $1.4 \mathrm{~mm}$ thick sample in frequency ranges of $5-8,8-12$, and $12-18 \mathrm{GHz}$. We find that $S_{21}$ is high, while $S_{11}$ is not higher than $5 \mathrm{~dB}$ for all spectra. Taking into account low $\mathrm{S}_{11}$, high $\mathrm{S}_{21}$ and power 
a
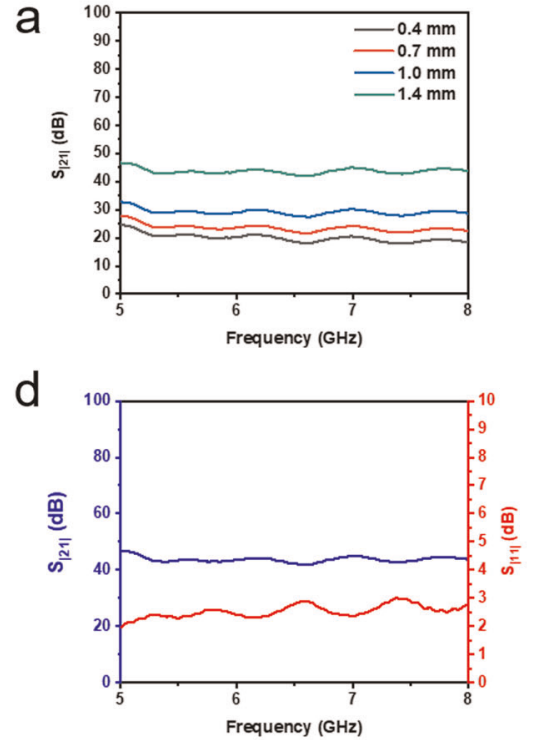

b

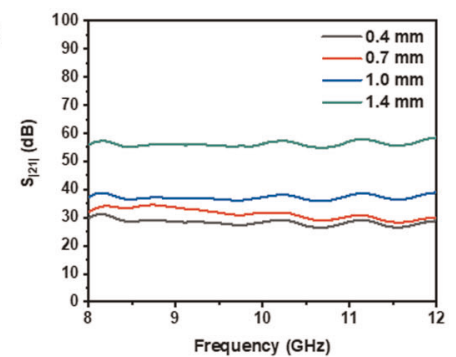

e

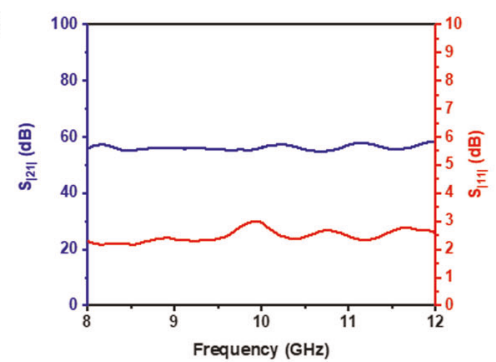

C

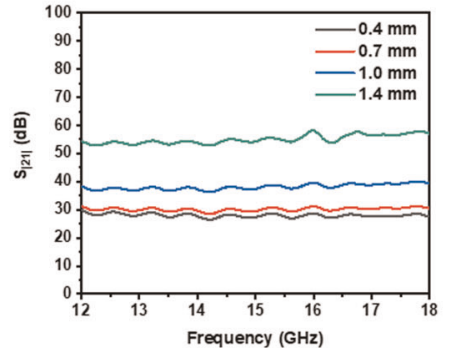

f

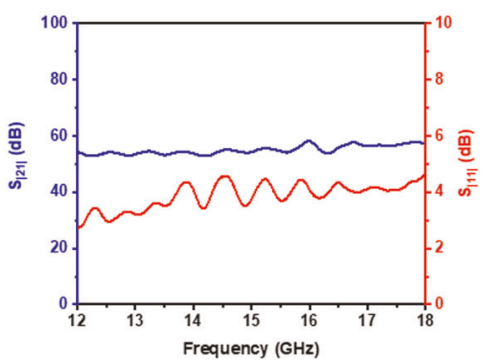

g

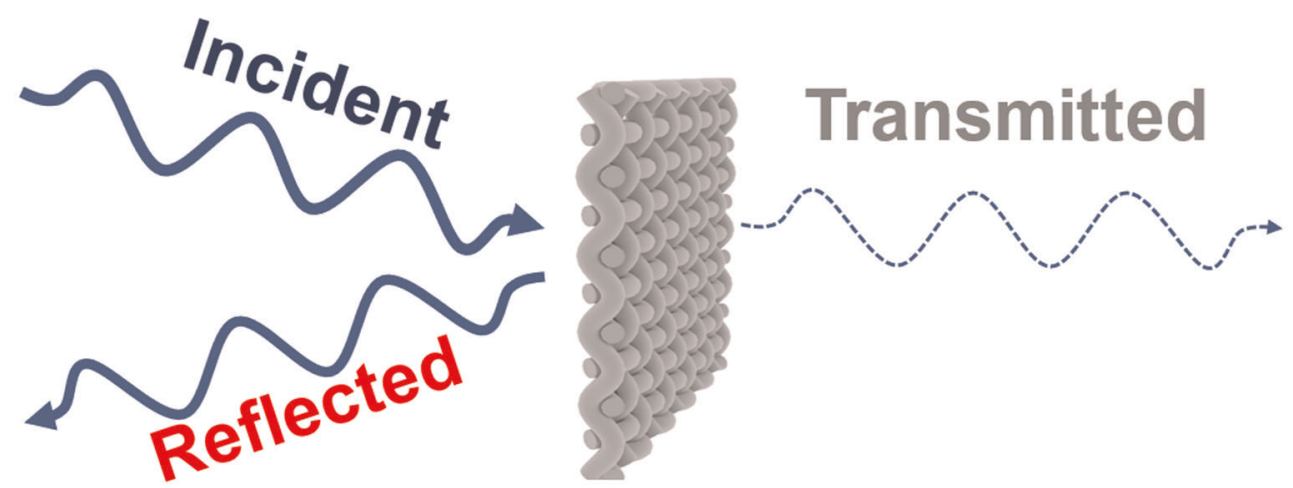

Fig. 3 Effectiveness of the EMI shielding and AgNWs/PVB textile schematic. a-c EMI SE AgNWs/PVB textile of various thickness in 5-8, 8-12, and $12-18 \mathrm{GHz}$ frequency ranges, including the S-parameter result of $\mathrm{S}_{21}$. d-f EMI SE of $1.4 \mathrm{~mm}$ thick AgNWs/PVB textile in 5-8, 8-12, and $12-18 \mathrm{GHz}$ frequency ranges, including the S-parameter result of $S_{21}$ (blue) and $S_{11}$ (red). $\mathbf{g}$ EMI shielding schematic of AgNWs/PVB textile including the incident, reflected and transmitted electromagnetic fields.

coefficient of reflectivity, transmissivity and absorptivity in Supplementary Note, we find that the principal mechanism of EMI SE is reflection rather than absorption, as shown by the schematic in Fig. 3g.

\section{Filling AgNWs/PVB textile in a jacket}

As a light and flexible EMI SE material, the AgNWs/PVB textile can be suitable for fitting into garments. However, it is necessary to test water and hot air resistance of the material beforehand. In order to measure water resistance, the AgNWs/PVB textile with and without PVB was immerged into deionized water for $48 \mathrm{~h}$, and then the content of $\mathrm{Ag}$ in water was measured via inductively coupled plasma (ICP). Figure 4a shows that fewer AgNWs fall off the textile substrate after PVB processing. Moreover, the water Ag content is high after washing the sample without PVB, which indicates that the PVB protects AgNWs from pilling off. In addition, we found that little variation in resistance appears when water is applied to the sample with PVB in comparison to the sample without PVB (Fig. 4b). The SEM image in Fig. 4c shows that after washing with water the AgNWs on the cotton skeleton remains stable with the PVB protector present. However, without the protection of PVB, the AgNWs fall off during washing and the network structure is broken (Supplementary Fig. 4). For testing heat resistance, we place the sample in hot air at $60^{\circ} \mathrm{C}$ and record the half-life of conductivity (Fig. 4d). It can be seen that the halflife of the sample with PVB exceeds one week while the half-life of the sample without PVB is below two days. It can be concluded that the PVB is essential for resistance to water and hot air. Finally, we place the large-area AgNWs/PVB textile material inside a garment (jacket). Figures $4 \mathrm{e}, \mathrm{f}$ show the schematic overview of the jacket. The EMI SE of the jacket was then measured by placing a mobile phone inside the jacket and measuring the electromagnetic field outside with a signal detector. It was found that the signal is significantly diminished when the phone is placed into a jacket with AgNWs/textile, while unchanged for the jacket without the AgNWs/PVB textile (Fig. 4g, h and Supplementary Video 1).

\section{DISCUSSION}

By employing an approach based on calculations and simulations, we designed and prepared a conductive network structure with high EMI SE via a facile immersing method employing AgNWs/PVB solution and textile. The AgNWs/PVB textile achieves a high EMI SE of $55 \mathrm{~dB}$ for a thickness of $1.4 \mathrm{~mm}$. Due to its very low density of 

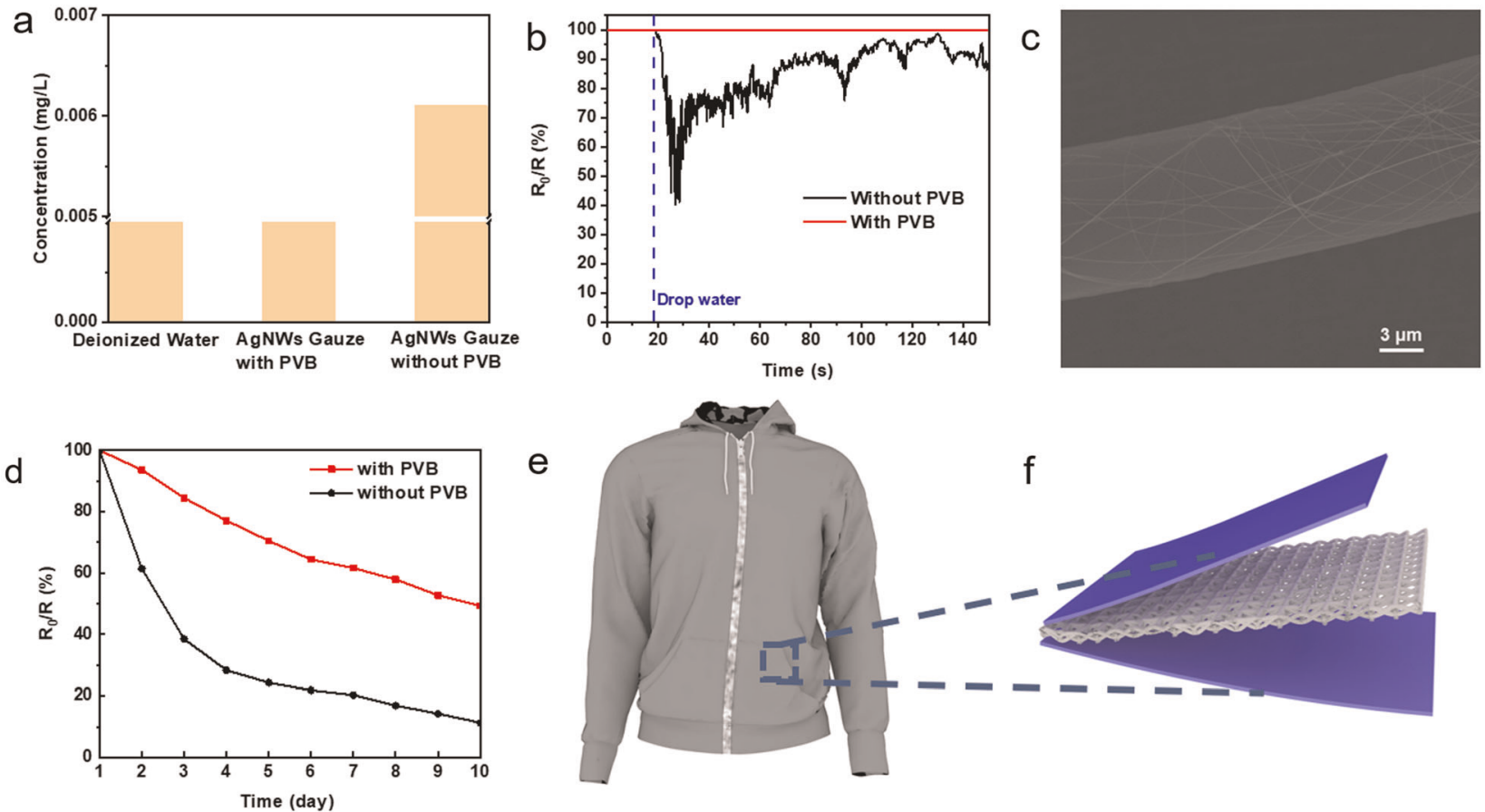

e
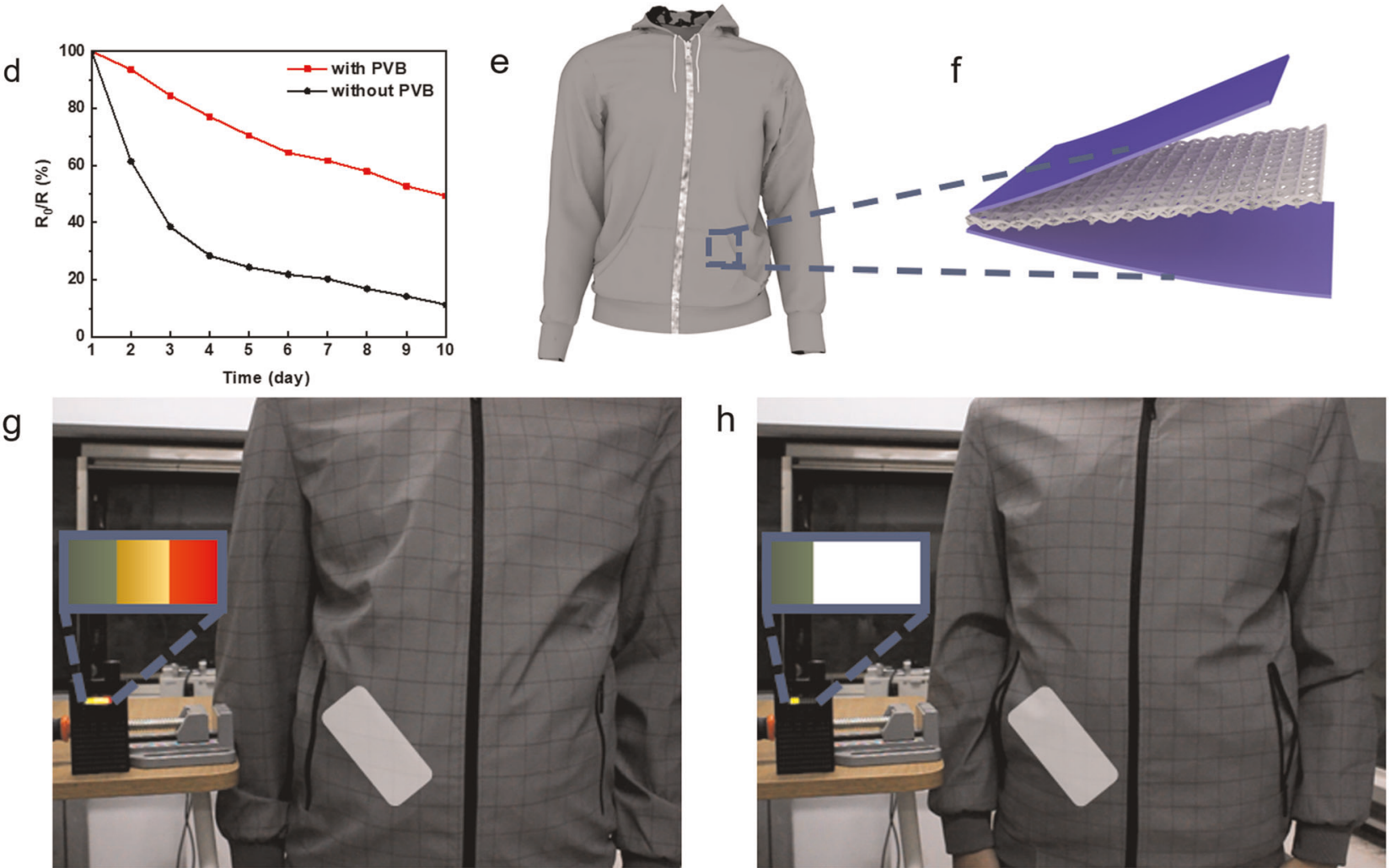

Fig. 4 Water washing and hot air resistance and example of application in an EMI shielding jacket. a The concentration of Ag in the water which is used to wash the AgNWs/PVB and AgNWs textile. $\mathbf{b}$ The resistive stability of AgNWs/PVB textile and AgNWs textile after moistened by water. c SEM image of a single skeleton of AgNWs/PVB textile after washing with water. $\mathbf{d}$ The changes in the resistance of AgNWs/PVB textile and AgNWs textile in $60^{\circ} \mathrm{C}$ hot air. e Schematic of a jacket with AgNWs/PVB textile and $\mathbf{f}$ the detail of every layer (the blues are the jacket shell fabric). A photograph with a magnification of the signal bar of a mobile phone signal detector with the phone placed inside the jacket pocket lined with AgNWs/PVB textile (g) and without AgNWs/PVB textile (h) (grey square denotes the position of the mobile phone).

$56 \mathrm{mg} / \mathrm{cm}^{3}$, the SSE reaches $1053 \mathrm{~dB} \cdot \mathrm{m}^{3} / \mathrm{g}$. In addition, the material is flexible and resistant to water washing and oxidation, and can be used in EMI shielding clothing. These unique properties make the AgNWs/PVB textile suitable for costeffective mass-produced EMI shielding materials.

\section{METHODS}

Simulation of the EMI SE effect

Time domain simulation was performed with Computer Simulation Technology Microwave Studio (CST MWS) software. The selected material was a metal with a conductivity of $630 \mathrm{~S} / \mathrm{m}^{2}$.

\section{Reagents}

Silver nanowires (AgNWs, $50 \mathrm{~nm}$ diameter, 100-200 nm length) (XFNANO Tech. Co., Ltd, Nanjing, China), polyvinyl butyral (PVB, M.W.= 170,000-250,000) ethanol (99.7\%) (Aladdin, USA), and textile were obtained commercially and used without further purification.

\section{Preparation of the EMI SE AgNWs/PVB textile}

In all, $150 \mathrm{mg}$ of PVB was dissolved in $30 \mathrm{~g}$ of ethanol and $1 \mathrm{~g} 20 \mathrm{mg} / \mathrm{ml}$ AgNWs ethanol was fully dispersed to obtain the PVB ethanol solution. A piece of textile was then immerged into the dispersion. Finally, the wet AgNWs/PVB textile was dried in a drying box at $60^{\circ} \mathrm{C}$ for $15 \mathrm{~min}$. The sample without PVB was prepared by same method with the PVB omitted.

\section{Material microscopic characterization}

The micro-morphological properties of AgNWs/PVB textile were measured with a field emission scanning electron microscope (FE-SEM, LEO-1530, Zeiss, Germany) and the micro-morphological properties of AgNWs were measured with a transmission electron microscope (TEM, JEOL-2100, JEOL Ltd. Japan). The crystal lattice parameters of samples were obtained with an X-ray diffractometer (XRD, D/max 2500, Rigaku, Japan) with $\mathrm{Cu} \mathrm{Ka}$ radiation $(\lambda=1.54178 \AA$ ). Thermogravimetric analysis and differential thermal gravity data were obtained with a thermogravimetric analyzer (STA 449 F3, Jupiter, Germany). XPS measurements were obtained with an $\mathrm{X}$-ray photoelectron spectrometer (Escalab 250Xi) equipped with an $\mathrm{Al} \mathrm{Ka}$ radiation source $(1487.6 \mathrm{eV})$ and hemispherical analyzer with pass energy 
of $30.0 \mathrm{eV}$ and a step size of $0.05 \mathrm{eV}$. The $\mathrm{C} 1 \mathrm{~s}$ peak at $284.8 \mathrm{eV}$ was taken as a reference for binding energy.

\section{Electronic EMI shielding characterization}

A CHI 760E electrochemical workstation (CHI Instrument, Shanghai, China) was used to test the stability of resistance with a stable voltage of $0.5 \mathrm{~V}$. EMI SE of AgNWs/PVB textile was measured with a vector network analyzer (VNA, E5063A 100kHz-18GHz, KEYSIGHT, USA). The cross-sectional area of $5-8,8-12$, and $12-18 \mathrm{GHz}$ waveguides were $34.849 \mathrm{~mm} \times 15.799 \mathrm{~mm}$, $22.86 \mathrm{~mm} \times 10.16 \mathrm{~mm}$ and $15.799 \mathrm{~mm} \times 7.899 \mathrm{~mm}$. The microwave was emitted from port 1 and the $S_{21}$ signal was received by port 2 . The $S_{11}$ signal is received by port 1 .

\section{DATA AVAILABILITY}

The authors declare that all data supporting the findings of this study are available within the paper and its supplementary information files.

Received: 28 January 2020; Accepted: 6 May 2020;

Published online: 01 June 2020

\section{REFERENCES}

1. Shahzad, F., Alhabeb, M., Hatter, C. B., Anasori, B. \& Hong, S. M. et al. Electromagnetic interference shielding with $2 \mathrm{D}$ transition metal carbides (MXenes). Science 353, 1137-40 (2016).

2. Lin, S., Wang, H., Wu, F., Wang, Q. \& Bai, X. et al. Room-temperature production of silver-nanofiber film for large-area, transparent and flexible surface electromagnetic interference shielding, npj Flexible. Electronics 3, 6 (2019).

3. Zhan, Y. H., Oliviero, M., Wang, J., Sorrentino, A. \& Buonocore, G. G. et al. Enhancing the EMI shielding of natural rubber-based supercritical $\mathrm{CO} 2$ foams by exploiting their porous morphology and CNT segregated networks. Nanoscale 11, 1011-20 (2019).

4. Fang, F., Li, Y. Q., Xiao, H. M., Hu, N. \& Fu, S. Y. Layer-structured silver nanowire/ polyaniline composite film as a high performance $\mathrm{X}$-band EMI shielding material. J. Mater. Chem. C. 4, 4193-203 (2016).

5. Al-Saleh, M. H. \& Sundararaj, U. X-band EMI shielding mechanisms and shielding effectiveness of high structure carbon black/polypropylene composites. J. Phys. D. Appl Phys. 46, 035304 (2013).

6. Singh, A. P., Garg, P., Alam, F., Singh, K. \& Mathur, R. et al. Phenolic resin-based composite sheets filled with mixtures of reduced graphene oxide, $\gamma$-Fe2O3 and carbon fibers for excellent electromagnetic interference shielding in the $\mathrm{X}$-band. Carbon 50, 3868-75 (2012).

7. El Kamchi, N., Belaabed, B., Wojkiewicz, J. L., Lamouri, S. \& Lasri, T. Hybrid polyaniline/nanomagnetic particles composites: High performance materials for EMI shielding. J. Appl Polym. Sci. 127, 4426-32 (2013).

8. Kim, J. T., Park, C. W. \& Kim, B. J. A study on synergetic EMI shielding behaviors of Ni-Co alloy-coated carbon fibers-reinforced composites. Synth. Met 223, 212-7 (2017).

9. Das, N. C., Liu, Y. Y., Yang, K. K., Peng, W. Q. \& Maiti, S. et al. Single-walled carbon nanotube/Poly(methyl methacrylate) composites for electromagnetic interference shielding. Polym. Eng. Sci. 49, 1627-34 (2009).

10. Chung, D. D. L. Electromagnetic interference shielding effectiveness of carbon materials. Carbon 39, 279-85 (2001).

11. Dalal, J., Lather, S., Gupta, A., Tripathi, R. \& Maan, A. S. et al. Reduced graphene oxide functionalized Strontium Ferrite in Poly (3, 4-ethylenedioxythiophene) conducting network: a high-performance EMI shielding material. Adv. Mater. Technol. 4, 1900023 (2019).

12. Luo, J. C., Wang, L., Huang, X. W., Li, B. \& Guo, Z. et al. Mechanically durable, highly conductive, and anticorrosive composite fabrics with excellent self-cleaning performance for high-efficiency electromagnetic interference shielding. Acs Appl. Mater. Inter, 11, 10883-94 (2019).

13. Zhu, S. P., Xing, C. T., Wu, F., Zuo, X. B. \& Zhang, Y. F. et al. Cake-like flexible carbon nanotubes/graphene composite prepared via a facile method for highperformance electromagnetic interference shielding. Carbon 145, 259-65 (2019).

14. Wang, Q. L., Xiao, S. L., Shi, S. Q., Xu, S. Y. \& Cai, L. P. Self-bonded natural fiber product with high hydrophobic and EMI shielding performance via magnetron sputtering Cu film. Appl. Surf. Sci. 475, 947-52 (2019).

15. Ghosh, S., Remanan, S., Mondal, S., Ganguly, S. \& Das, P. et al. An approach to prepare mechanically robust full IPN strengthened conductive cotton fabric for high strain tolerant electromagnetic interference shielding. Chem. Eng. J. 344, 138-54 (2018).
16. Ghosh, S., Mondal, S., Ganguly, S., Remanan, S. \& Singha, N. et al. Carbon nanostructures based mechanically robust conducting cotton fabric for improved electromagnetic interference shielding. Fiber Polym. 19, 1064-73 (2018).

17. Ghosh, S., Ganguly, S., Das, P., Das, T. K. \& Bose, M. et al. Fabrication of reduced graphene oxide/silver nanoparticles decorated conductive cotton fabric for high performing electromagnetic interference shielding and antibacterial application. Fiber Polym. 20, 1161-71 (2019).

18. Ram, R., Khastgir, D. \& Rahaman, M. Electromagnetic interference shielding effectiveness and skin depth of poly (vinylidene fluoride)/particulate nanocarbon filler composites: prediction of electrical conductivity and percolation threshold. Polym. Int. 68, 1194-203 (2019).

19. Yang, H., Bai, S. C., Guo, X. Z. \& Wang, H. F. Robust and smooth UV-curable layer overcoated AgNW flexible transparent conductor for EMI shielding and film heater. Appl. Surf. Sci. 483, 888-94 (2019).

20. Kong, L., Yin, X. W., Xu, H. L., Yuan, X. Y. \& Wang, T. et al. Powerful absorbing and lightweight electromagnetic shielding CNTs/RGO composite. Carbon 145, 61-6 (2019).

21. Huang, L., Li, J. J., Li, Y. B., Heb, X. D. \& Yuan, Y. Lightweight and flexible hybrid film based on delicate design of electrospun nanofibers for high-performance electromagnetic interference shielding. Nanoscale 11, 8616-25 (2019).

22. Lin, S. F., Ju, S., Shi, G., Zhang, J. W. \& He, Y. L. et al. Ultrathin nitrogen-doping graphene films for flexible and stretchable EMI shielding materials. J. Mater. Sci. 54, 7165-79 (2019).

23. Ghosh, S., Ganguly, S., Remanan, S. \& Das, N. C. Fabrication and investigation of 3D tuned PEG/PEDOT: PSS treated conductive and durable cotton fabric for superior electrical conductivity and flexible electromagnetic interference shielding. Compos. Sci. Technol. 181, 107682 (2019).

24. Ravindren, R., Mondal, S., Nath, K. \& Das, N. C. Synergistic effect of double percolated co-supportive MWCNT-CB conductive network for high-performance EMI shielding application. Polym. Adv. Technol. 30, 1506-17 (2019).

25. Zhang, X. L., Zhang, X. M., Yang, M. T., Yang, S. \& Wu, H. et al. Ordered multilayer film of (graphene oxide/polymer and boron nitride/polymer) nanocomposites: an ideal EMI shielding material with excellent electrical insulation and high thermal conductivity. Compos. Sci. Technol. 136, 104-10 (2016).

26. Pawar, S. P., Rzeczkowski, P., Potschke, P., Krause, B. \& Bose, S. Does the processing method resulting in different states of an interconnected network of multiwalled carbon nanotubes in polymeric blend nanocomposites affect EMI shielding properties? ACS Omega 3, 5771-82 (2018).

27. Ghosh, S., Das, P., Ganguly, S., Remanan, S. \& Das, T. K. et al. 3D-Enhanced, highperforming, super-hydrophobic and electromagnetic-interference shielding fabrics based on silver paint and their use in antibacterial applications. Chemistryselect 4, 11748-54 (2019).

28. Lin, S., Wang, H., Zhang, X., Wang, D. \& Zu, D. et al. Direct spray-coating of highly robust and transparent $\mathrm{Ag}$ nanowires for energy saving windows. Nano Energy 62, 111-6 (2019).

29. Lin, S., Bai, X., Wang, H., Wang, H. \& Song, J. et al. Roll-to-roll production of transparent silver-nanofiber-network electrodes for flexible electrochromic smart windows. Adv. Mater. 29, 1703238 (2017).

30. Wang, J. L., Hassan, M., Liu, J. W. \& Yu, S. H. Nanowire assemblies for flexible electronic devices: recent advances and perspectives. Adv. Mater. 30, 1803430 (2018).

31. Choi, S., Han, S. I., Jung, D., Hwang, H. J. \& Lim, C. et al. Highly conductive, stretchable and biocompatible $\mathrm{Ag}-\mathrm{Au}$ core-sheath nanowire composite for wearable and implantable bioelectronics. Nat. Nanotechnol. 13, 1048-1056 (2018).

32. Lee, S. H., Yu, S., Shahzad, F., Hong, J. P. \& Kim, W. N. et al. Highly anisotropic Cu oblate ellipsoids incorporated polymer composites with excellent performance for broadband electromagnetic interference shielding. Compos. Sci. Technol. 144, 57-62 (2017).

33. Ma, J. J., Zhan, M. S. \& Wang, K. Ultralightweight silver nanowires hybrid polyimide composite foams for high-performance electromagnetic interference shielding. ACS Appl Mater. Interfaces 7, 563-76 (2015).

34. Liu, W., Liu, M., Lin, S., Liu, J. \& Lei, M. et al. Synthesis of high quality silver nanowires and their applications in ultrafast photonics. Opt. Express 27, 16440-8 (2019).

35. Lin, S., Liu, J. C., Li, W. Z., Wang, D. \& Huang, Y. et al. A flexible, robust, and gel-free electroencephalogram electrode for noninvasive brain-computer interfaces. Nano Lett. 19, 6853-61 (2019).

36. Bayat, M., Yang, H., Ko, F. K., Michelson, D. \& Mei, A. Electromagnetic interference shielding effectiveness of hybrid multifunctional Fe304/carbon nanofiber composite. Polymer 55, 936-43 (2014).

37. Al-Saleh, M. H. \& Sundararaj, U. A review of vapor grown carbon nanofiber/ polymer conductive composites. Carbon 47, 2-22 (2009).

38. Ge, J., Wang, X., Yao, H. B., Zhu, H. W. \& Peng, Y. C. et al. Durable $\mathrm{Ag} / \mathrm{AgCl}$ nanowires assembled in a sponge for continuous water purification under sunlight. Mater. Horiz. 2, 509-13 (2015). 
39. Daoud, W. A. \& Xin, J. H. Nucleation and growth of anatase crystallites on cotton fabrics at low temperatures. J. Am. Ceram. Soc. 87, 953-5 (2004).

40. Topalovic, T., Nierstrasz, V. A., Bautista, L., Jocic, D. \& Navarro, A. et al. XPS and contact angle study of cotton surface oxidation by catalytic bleaching. Colloids Surf. A: Physicochem. Eng. Asp. 296, 76-85 (2007).

\section{ACKNOWLEDGEMENTS}

This work was financially supported by National Natural Science Foundation of China (Grant 61672108, 61874014), China Postdoctoral Science Foundation (2018M640124, 2019T120083) and Program for Fund of State Key Laboratory of Information Photonics and Optical Communications (Beijing University of Posts and Telecommunications, China).

\section{AUTHOR CONTRIBUTIONS}

H.W. and J.C.L. conceived the idea and designed the research. J.C.L., S.L., C.J., prepared the materials. J.C.L., S.L., H.Y.W., J.A.S., Z.L.L. design the production technology. J.C.L., S.L., conducted the micromorphological characterization of material, including XRD, SEM, TEM, XPS, and TGA. J.C.L., S.L., conducted the EMI SE measurement and analyzed the EMI SE data. J.C.L., Q.M.W conducted the simulation and theoretical calculation J.C.L., S.L., Z.W.L, H.Y.W., M.L. conducted the visible-light photography and performance of an EMI shielding jacket. J.C.L., S.L., conducted the resistant tests. All authors contributed to paper writing. J.C.L and S.L. are co-first authors.

\section{COMPETING INTERESTS}

The authors declare no competing interests.

\section{ADDITIONAL INFORMATION}

Supplementary information is available for this paper at https://doi.org/10.1038/ s41528-020-0074-0.

Correspondence and requests for materials should be addressed to M.L. or H.W.

Reprints and permission information is available at http://www.nature.com/ reprints

Publisher's note Springer Nature remains neutral with regard to jurisdictional claims in published maps and institutional affiliations.

Open Access This article is licensed under a Creative Commons Attribution 4.0 International License, which permits use, sharing, adaptation, distribution and reproduction in any medium or format, as long as you give appropriate credit to the original author(s) and the source, provide a link to the Creative Commons license, and indicate if changes were made. The images or other third party material in this article are included in the article's Creative Commons license, unless indicated otherwise in a credit line to the material. If material is not included in the article's Creative Commons license and your intended use is not permitted by statutory regulation or exceeds the permitted use, you will need to obtain permission directly from the copyright holder. To view a copy of this license, visit http://creativecommons. org/licenses/by/4.0/.

(c) The Author(s) 2020 